\title{
Spin Crossover in $\mathrm{Cu}_{x} \mathrm{Co}_{y} \mathrm{Cr}_{z} \mathrm{Se}_{4}$ Semiconductors
}

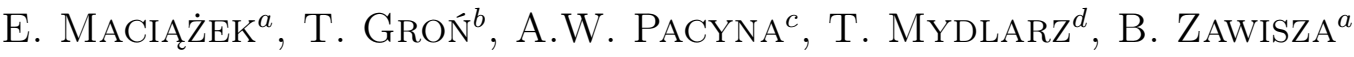 \\ AND J. KROK-KOWALSKI ${ }^{b}$ \\ ${ }^{a}$ Institute of Chemistry, University of Silesia, Szkolna 9, 40-006 Katowice, Poland \\ ${ }^{b}$ Institute of Physics, University of Silesia, Uniwersytecka 4, 40-007 Katowice, Poland \\ ${ }^{c}$ The Henryk Niewodniczański Institute of Nuclear Physics, Polish Academy of Sciences \\ E. Radzikowskiego 152, 31-342 Kraków, Poland \\ ${ }^{d}$ International Laboratory of High Magnetic Fields and Low Temperatures \\ Gajowicka 95, 53-529 Wrocław, Poland
}

\begin{abstract}
Magnetization and magnetic susceptibility measured in the zero-field-cooled mode were used to study the spin crossover transition in polycrystalline $\mathrm{Cu}_{x} \mathrm{Co}_{y} \mathrm{Cr}_{z} \mathrm{Se}_{4}$ compounds. With increasing Co content a transition from ferromagnetic order via ferrimagnetic one to antiferromagnetic-like behaviour was observed. This transition is accompanied with a lowering symmetry from cubic to monoclinic and for the latter the spin crossover phenomenon occurs. These results are considered in a framework of the ligand-field split and the spin-orbit coupling.
\end{abstract}

PACS: 75.30.Wx, 75.50.Dd, 75.50.Ee

\section{Introduction}

Structural and electrical investigations carried out on the polycrystalline $\mathrm{Cu}_{1-x} \mathrm{Co}_{x} \mathrm{Cr}_{2} \mathrm{Se}_{4}$ compounds in the compositional range $0.0 \leq x \leq 1.0$ revealed semiconducting properties and a transformation from cubic spinel-type to monoclinic $\mathrm{Cr}_{3} \mathrm{~S}_{4}$-type structure above $x=$ 0.4 [1]. Recently, the X-ray diffraction studies on the polycrystalline $\mathrm{Cu}_{1-x} \mathrm{Co}_{x} \mathrm{Cr}_{2} \mathrm{Se}_{4}$ system $(x=0.0,0.2$, $0.4,0.6,0.8,1.0)$ proved the existence of the cubic spinel-type structure for $x \leq 0.2$ and the monoclinic $\mathrm{Cr}_{3} \mathrm{~S}_{4}$-type one for $x \geq 0.8$ [2]. $\mathrm{CoCr}_{2} \mathrm{Se}_{4}$ undergoes an order-disorder phase transition above $750{ }^{\circ} \mathrm{C}$ [2] and has a sharp peak in its susceptibility at $199 \mathrm{~K}$ suggesting antiferromagnetic (AFM) behaviour [3]. Electrical and magnetic studies carried out on single-crystalline non-stoichiometric $\mathrm{Cu}_{x} \mathrm{Co}_{y} \mathrm{Cr}_{z} \mathrm{Se}_{4}$ spinels showed ferromagnetic (FM) ordering and $p$-type metallic conductivity for $y=0.06,0.1$ and 0.11 as well as a ferrimagnetic behaviour and $n$-type electrical semiconductivity for $y=0.23[4]$.

It is well known in literature that compounds containing transition metals with $d^{4}, d^{5}, d^{6}$, and $d^{7}$ electron configurations usually are capable of forming either spin-free (high-spin, HS) or spin-paired (low-spin, LS) grounds states, depending on the strength of the cubic ligand field, $\Delta$, relative to the mean spin-pairing energy, $\bar{P}[5]$. Then, their magnetism follows the Curie-Weiss law. However, when $|\Delta-\bar{P}|$ becomes comparable to thermal energy, $k T$, both spin states HS and LS may be populated and cross over in thermal equilibrium. This phenomenon is commonly known as spin crossover for that the Curie-Weiss law is not valid. So, the easiest way of following the spin conversion as a function of temperature is the measurement of the magnetic susceptibility [5].

It seems thus natural to expect a spin transition in the polycrystalline $\mathrm{Cu}_{x} \mathrm{Co}_{y} \mathrm{Cr}_{z} \mathrm{Se}_{4}$ system for higher Co content.

\section{Experimental details}

Powder samples of the $\mathrm{Cu}_{x} \mathrm{Co}_{y} \mathrm{Cr}_{z} \mathrm{Se}_{4}$ system were obtained as ceramics using a preparation method which is described in detail elsewhere [1]. Chemical composition of the compounds was determined by energy-dispersive X-ray fluorescence (EDXRF) spectrometry. The samples were excited by the Rh target X-ray tube (XTF 5011/ 75 , Oxford Instruments, USA). The X-ray spectra of the samples were collected by thermoelectrically cooled Si-PIN detector (XR-100CR Amptek, Bedford, MA, USA) of $145 \mathrm{eV}$ resolution at $5.9 \mathrm{keV}$. The EDXRF spectrometer is described in detail in Ref. [6]. The quantitative EDXRF analysis of samples was performed by fundamental parameters method based on the Sherman equation. The compositions studied in this paper are the same as reported in Ref. [1].

Magnetization and magnetic susceptibility were measured in the zero-field-cooled mode using a vibrating sample magnetometer with a step motor [7] at $4.2 \mathrm{~K}$ and in applied external magnetic fields up to $150 \mathrm{kOe}$ and a Faraday type Cahn RG automatic electrobalance in the temperature range $4.2-400 \mathrm{~K}$ and at $800 \mathrm{Oe}$, respectively. 


\section{Results and discussion}

Figure 1 shows the magnetization isotherms measured at $4.2 \mathrm{~K}$. The spinel samples with low Co content up to $y=0.25$ reach full saturation of $4.65 \mu_{\mathrm{B}}$ /f.u. for $\mathrm{Cu}_{0.93} \mathrm{Co}_{0.15} \mathrm{Cr}_{1.64} \mathrm{Se}_{4}$ and of $4.35 \mu_{\mathrm{B}} /$ f.u. for $\mathrm{Cu}_{0.75} \mathrm{Co}_{0.25} \mathrm{Cr}_{2} \mathrm{Se}_{4}$ at $140 \mathrm{kOe}$ close to the theoretical value of $5 \mu_{\mathrm{B}}$ per molecule for $\mathrm{Cu}\left[\mathrm{Cr}^{3+} \mathrm{Cr}^{4+}\right] \mathrm{Se}_{4}$ spinel [8]. In these spinels the magnetic moments order ferromagnetically and the Curie temperature $T_{\mathrm{C}}$ decreases from $390 \mathrm{~K}$ for $y=0.15$ to $377 \mathrm{~K}$ for $y=0.25$ (see Fig. 2). A pronounced decrease of saturation moment with increasing Co content suggests the LS state of the $\mathrm{Co}^{2+}$ ions, tetrahedrally coordinated, with spin $S=1 / 2$ in the $t_{2}$ orbital (see Fig. 3 ), because the $\mathrm{Cr}^{3+}$ ions, octahedrally coordinated, with spin $S=3 / 2$ in the $t_{2 \mathrm{~g}}$ orbital are always in the HS state. Eventually, a FM and AFM coupling between $\mathrm{Co}^{2+}$ and $\mathrm{Cr}^{3+}$ magnetic moments may occur, too.

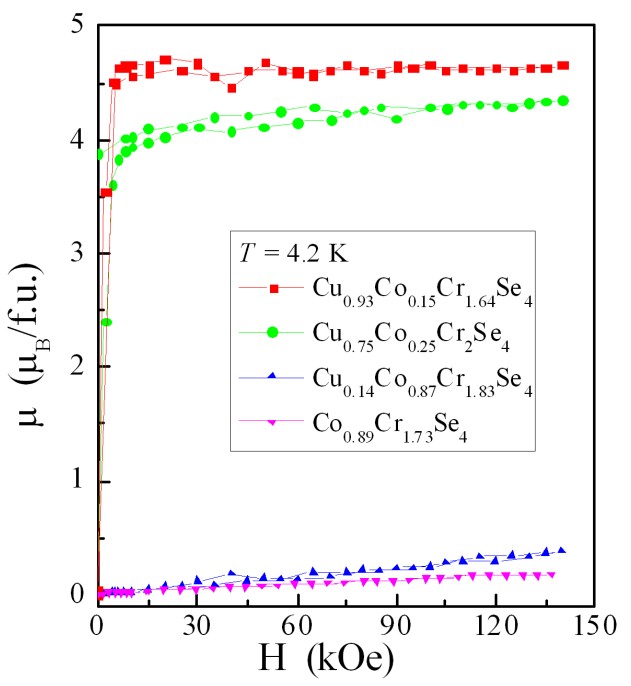

Fig. 1. Magnetic moment $\mu$ vs. magnetic field $H$ for the $\mathrm{Cu}_{x} \mathrm{Co}_{y} \mathrm{Cr}_{z} \mathrm{Se}_{4}$ system at $4.2 \mathrm{~K}$.

The monoclinic $\mathrm{Cr}_{3} \mathrm{~S}_{4}$-type structures with high Co content above $y=0.8$ show a dramatically strong drop of magnetic moment up to $0.18 \mu_{\mathrm{B}} /$ f.u. at $137 \mathrm{kOe}$ for $y=0.89$. They do not reveal both saturation and spontaneous magnetization (Fig. 1). The temperature dependence of magnetic susceptibility, $\chi_{\sigma}(T)$, depicted in Fig. 4 reveals ferrimagnetic order with $T_{\mathrm{C}}=133 \mathrm{~K}$ and without a Curie-Weiss behavior in the temperature range $4.2-400 \mathrm{~K}$ for $\mathrm{Cu}_{0.14} \mathrm{Co}_{0.87} \mathrm{Cr}_{1.83} \mathrm{Se}_{4}$. Above $T_{\mathrm{C}}$ the $\chi_{\sigma}(T)$ curve shows minimum at $T_{1}=168 \mathrm{~K}$ and maximum at $T_{2}=255 \mathrm{~K}$ characteristic for a spin crossover transition. The non-linear dependence of $1 / \chi_{\sigma}(T)$ at 800 Oe maybe a consequence of the strong dependence of the effective magnetic moment with temperature, which is experimentally observed in the compounds where the spin crossover phenomenon occurs [5]. Similar behavior is observed for $\mathrm{Co}_{0.89} \mathrm{Cr}_{1.73} \mathrm{Se}_{4}$ depicted in Fig. 5. The temperature dependence of magnetic susceptibility, $\chi_{\sigma}(T)$,

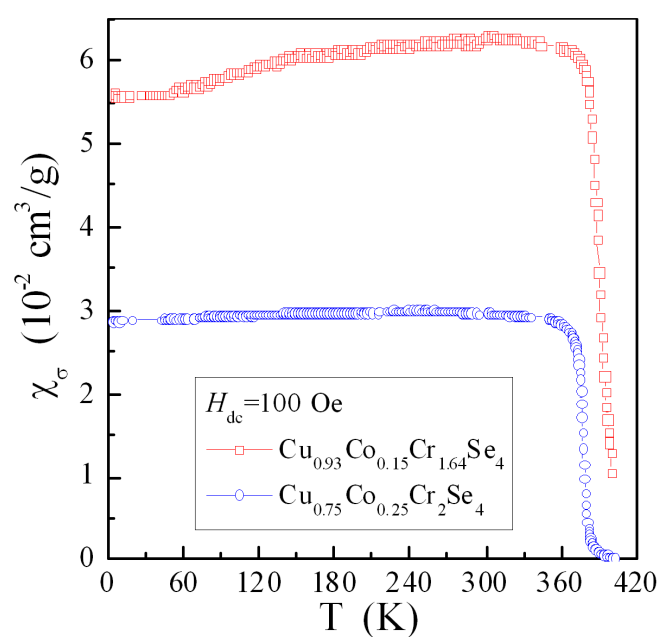

Fig. 2. The ZFC dc mass susceptibility $\chi_{\sigma}$ vs. temperature $T$ for polycrystalline $\mathrm{Cu}_{0.93} \mathrm{Co}_{0.15} \mathrm{Cr}_{1.64} \mathrm{Se}_{4}$ and $\mathrm{Cu}_{0.75} \mathrm{Co}_{0.25} \mathrm{Cr}_{2} \mathrm{Se}_{4}$ ferromagnetic spinels recorded at $H_{\mathrm{dc}}=100 \mathrm{Oe}$.

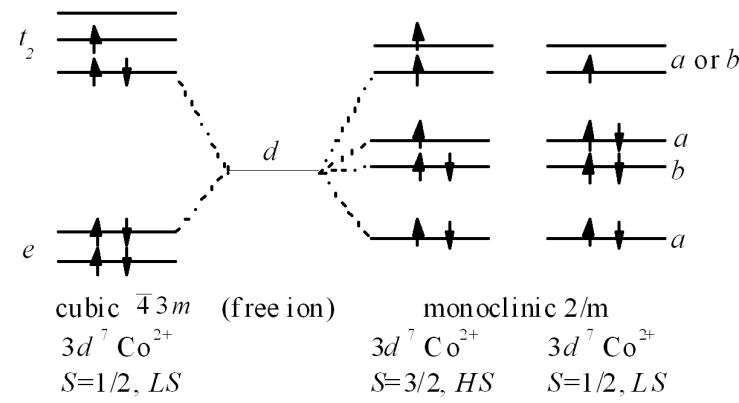

Fig. 3. The ligand-field split components showing the symmetry-lowered phase transitions in $\mathrm{Cu}_{x} \mathrm{Co}_{y} \mathrm{Cr}_{z} \mathrm{Se}_{4}$ for high Co content $(y \geq 0.8)$.

reveals antiferromagnetic-like behaviour with $T_{\mathrm{N}}=6 \mathrm{~K}$ and without a Curie-Weiss behavior in the temperature range $4.2-400 \mathrm{~K}$. Above $T_{\mathrm{N}}$ the $\chi_{\sigma}(T)$ curve shows minimum at $T_{1}=118 \mathrm{~K}$ and maximum at $T_{2}=286 \mathrm{~K}$.

These results seem to be connected with thermally induced LS-HS transitions in the $\mathrm{Cu}_{x} \mathrm{Co}_{y} \mathrm{Cr}_{z} \mathrm{Se}_{4}$ system for $y \geq 0.8$. For the $\mathrm{Co}^{2+}$ ion with $3 d^{7}$ electronic configurations the multiplet width should be comparable to $k T$ because the temperature dependence of magnetic susceptibility, described by the Curie-Weiss law is not obeyed and the magnetic moment is strongly lowered. Only a slight decrease in magnetic moment could be related to the magnetic moment of $\mathrm{Co}$, which would be perpendicular to the magnetic moment of $\mathrm{Cr}$. It takes into the effect that the longitudinal component of the moment of the Co ions becomes zero and only the Cr moment remains visible in the saturation state, as it was found for $\mathrm{MnCr}_{2} \mathrm{~S}_{4}$ [9] and $\mathrm{Mn}\left[\mathrm{Cr}_{0.5} \mathrm{Ga}_{1.5}\right] \mathrm{S}_{4}$ [10].

At $T_{2}=255 \mathrm{~K}$ for $\mathrm{Cu}_{0.14} \mathrm{Co}_{0.87} \mathrm{Cr}_{1.83} \mathrm{Se}_{4}$ and at $T_{2}=286 \mathrm{~K}$ for $\mathrm{Co}_{0.89} \mathrm{Cr}_{1.73} \mathrm{Se}_{4}$ thermal energy $k T_{2}=$ 


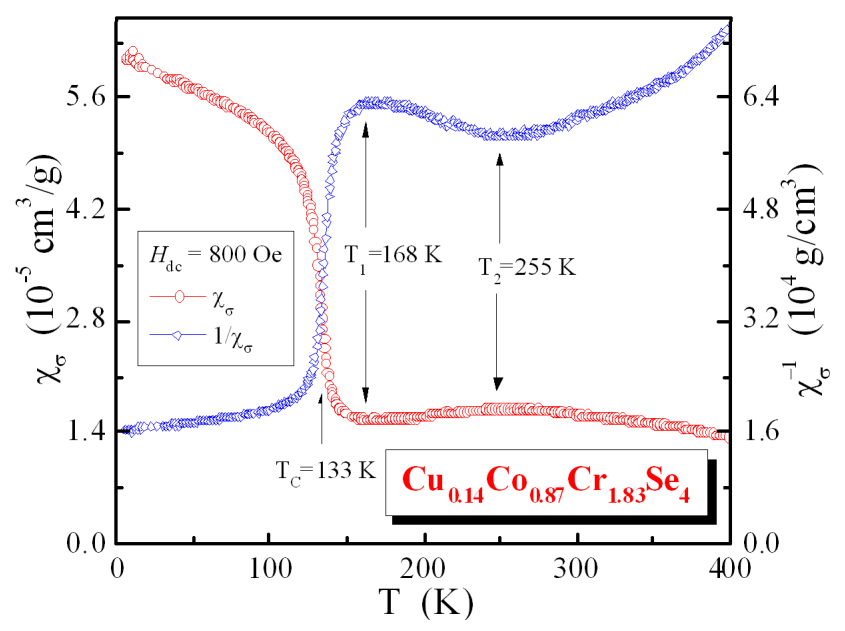

Fig. 4. The ZFC dc mass susceptibility $\chi_{\sigma}$ and $1 / \chi_{\sigma}$ vs. temperature $T$ for $\mathrm{Cu}_{0.14} \mathrm{Co}_{0.87} \mathrm{Cr}_{1.83} \mathrm{Se}_{4}$ recorded at $H_{\mathrm{dc}}=800$ Oe. $T_{\mathrm{C}}$ is the Curie temperature and $T_{1}$ and $T_{2}$ are temperatures characteristic for spin crossover transition.

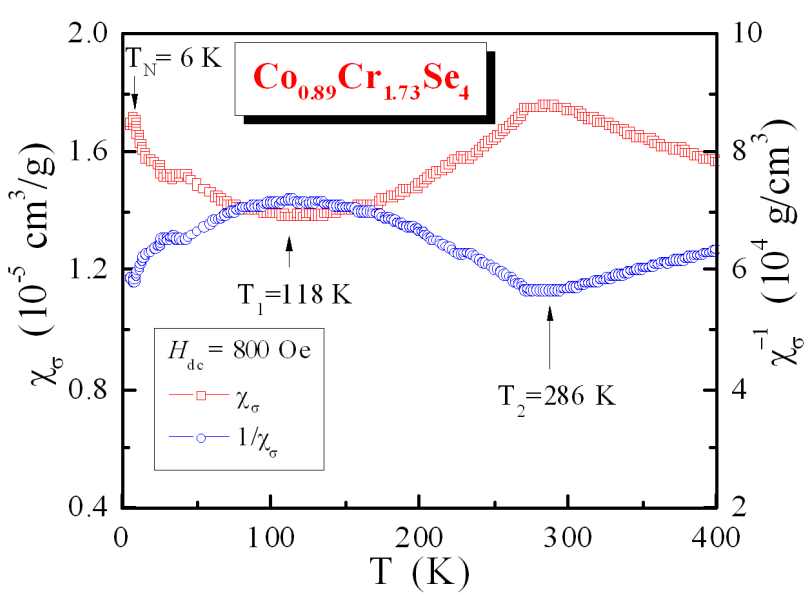

Fig. 5. The ZFC dc mass susceptibility $\chi_{\sigma}$ and $1 / \chi_{\sigma}$ vs. temperature $T$ for $\mathrm{Co}_{0.89} \mathrm{Cr}_{1.73} \mathrm{Se}_{4}$ recorded at $H_{\mathrm{dc}}=800$ Oe. $T_{\mathrm{N}}$ is the Néel temperature and $T_{1}$ and $T_{2}$ are temperatures characteristic for spin crossover transition.

$0.0213 \mathrm{eV}$ and $0.0238 \mathrm{eV}$, respectively. These energies are over 20 times smaller in comparison with the tetrahedral orbital splitting of $\mathrm{Co}^{2+}$ ion, $\Delta_{\mathrm{t}}=0.5514 \mathrm{eV}$. A lowering symmetry, displayed in Fig. 3 , from cubic $(\overline{4} 3 \mathrm{~m})$ to monoclinic $(2 / m)$ [11] makes easier the spin crossover transitions as the orbital splitting is lowered giving the value $|\Delta-\bar{P}|$ comparable to $k T$. We suggest that the broad humps on the $\chi_{\sigma}(T)$ curves above the ordering temperature may correspond to the ${ }^{4} T \leftrightarrow{ }^{2} E$ spin crossover in cobalt(II).

\section{Conclusions}

We have observed an anomalous behaviour of the temperature dependence of magnetic susceptibility and magnetization of the $\mathrm{Cu}_{x} \mathrm{Co}_{y} \mathrm{Cr}_{z} \mathrm{Se}_{4}$ compounds for high cobalt content $(y \geq 0.8)$ in comparison with low cobalt one $(y \leq 0.25)$. The high content of the Co-ions weakens the FM long-range interactions. Therefore, with increasing Co content a transition from ferromagnetic order via ferrimagnetic one to antiferromagnetic-like behaviour is observed. This transition is accompanied with a lowering symmetry from cubic $(\overline{4} 3 \mathrm{~m})$ to monoclinic $(2 / \mathrm{m})$ and for the latter the spin crossover phenomenon occurs. The broad humps at $255 \mathrm{~K}$ for $\mathrm{Cu}_{0.14} \mathrm{Co}_{0.87} \mathrm{Cr}_{1.83} \mathrm{Se}_{4}$ and at $286 \mathrm{~K}$ for $\mathrm{Co}_{0.89} \mathrm{Cr}_{1.73} \mathrm{Se}_{4}$ on the $\chi_{\sigma}(T)$ curves above the ordering temperature are characteristic for the HS-LS transitions. The nature of these transitions seems to be connected with the spin-orbit coupling giving the multiplet width comparable to thermal energy.

\section{Acknowledgments}

This work is partly founded from science grant No. N N204 289134.

\section{References}

[1] E. Maciążek, A. Molak, T. Goryczka, J. Alloys Comp. 441, 222 (2007).

[2] V. Svitlyk, Y. Mozharrivskyj, Inorg. Chem. 48, 5296 (2009).

[3] B.L. Morris, P. Russo, A. Wold, J. Phys. Chem. Solids 31, 635 (1970).

[4] T. Groń, E. Maciążek, J. Heimann, J. Kusz, I. Okońska-Kozłowska, K. Bärner, and Ch. Kleeberg, Physica B 254, 84 (1998).

[5] P. Gütlich, Spin Crossover in Iron(II)-Complexes in Structure $\&$ Bonding, Vol. 44, Springer, Berlin 1981.

[6] R. Sitko, B. Zawisza, E. Malicka, Spectrochim. Acta B 64, 436 (2009).

[7] V.I. Nizhankovskii, L.B. Lugansky, Meas. Sci. Technol. 18, 1533 (2007).

[8] O. Yamashita, Y. Yamaguchi, I. Nakatani, H. Watanabe, K. Masumoto, J. Phys. Soc. Jpn. 46, 1145 (1979).

[9] V. Tsurkan, M. Mücksch, V. Fritsch, J. Hemberger, M. Klemm, S. Klimm, S. Körner, H.-A. Krug, D. von Nidda, E.-W. Samusi, A. Scheidt, S. Loidl, R. Horn, Tidecks, Phys. Rev. B 68, 134434 (2003).

[10] T. Groń, J. Krok-Kowalski, A.W. Pacyna, T. Mydlarz, J. Phys. Chem. Solids 70, 900 (2009).

[11] C.F. van Bruggen, Ann. Chim. Fr. 7, 171 (1982). 